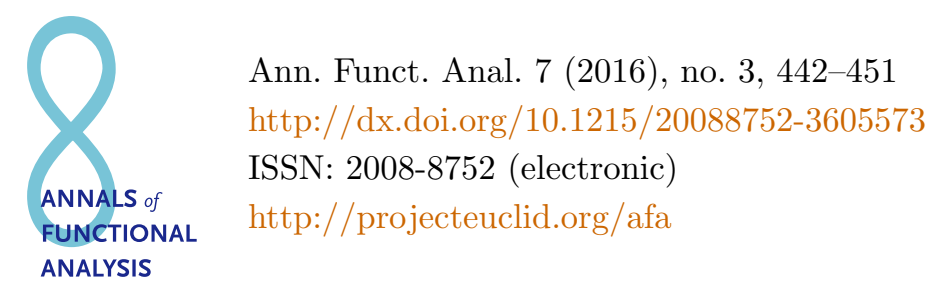

\title{
DISJOINTNESS-PRESERVING LINEAR MAPS ON BANACH FUNCTION ALGEBRAS ASSOCIATED WITH A LOCALLY COMPACT GROUP
}

\author{
J. ALAMinOS, J. EXTREMERA, and A. R. VILLENA* \\ Communicated by K. F. Taylor
}

\begin{abstract}
We introduce a certain property of commutative Banach algebras which we call property $\mathrm{OB}$. We prove that every bounded disjointnesspreserving linear map from a commutative Banach algebra with the aforesaid property to any semisimple, commutative Banach algebra is a weighted composition map. Further, it is shown that a variety of important Banach algebras in harmonic analysis have the property $\mathrm{OB}$.
\end{abstract}

\section{INTRODUCTION}

The basic aim of this article is to bring together the theory of operator hyperTauberian Banach algebras developed by Samei in [15] and the pattern established in [1] with the purpose of analyzing the so-called disjointness-preserving linear maps. This class of maps has been extensively studied in different contexts: Banach lattices, function algebras, and general Banach algebras. A linear map $\Phi: A \rightarrow B$ between Banach function algebras $A$ and $B$ is said to be disjointness-preserving if $\Phi(a) \Phi(b)=0$ whenever $a, b \in A$ are such that $a b=0$. The question of whether such a map for certain algebras is a weighted composition map has been widely studied. This paper focuses on a variety of significant Banach function algebras associated with a locally compact group $G$ such as the Figà-Talamanca-Herz algebra $A_{p}(G)$ and the Figà-Talamanca-Herz-Lebesgue

Copyright 2016 by the Tusi Mathematical Research Group.

Received Oct. 8, 2015; Accepted Jan. 18, 2016.

${ }^{*}$ Corresponding author.

2010 Mathematics Subject Classification. Primary 43A15; Secondary 43A30, 46J10.

Keywords. Fourier algebra, Figà-Talamanca-Herz algebra, Figà-Talamanca-Herz-Lebesgue algebra, operator hyper-Tauberian Banach algebra, disjointness-preserving linear map. 


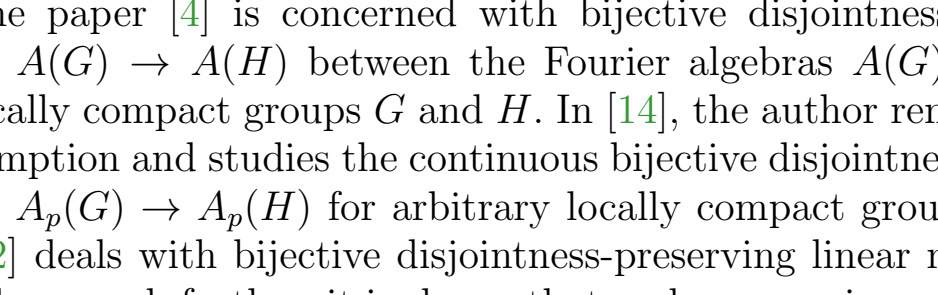

\title{
DISJOINTNESS-PRESERVING LINEAR MAPS ON BANACH FUNCTION ALGEBRAS ASSOCIATED WITH A LOCALLY COMPACT GROUP
}

\author{
J. ALAMinOS, J. EXTREMERA, and A. R. VILLENA* \\ Communicated by K. F. Taylor
}

\begin{abstract}
We introduce a certain property of commutative Banach algebras which we call property $\mathrm{OB}$. We prove that every bounded disjointnesspreserving linear map from a commutative Banach algebra with the aforesaid property to any semisimple, commutative Banach algebra is a weighted composition map. Further, it is shown that a variety of important Banach algebras in harmonic analysis have the property $\mathrm{OB}$.
\end{abstract}

\section{INTRODUCTION}

The basic aim of this article is to bring together the theory of operator hyperTauberian Banach algebras developed by Samei in [15] and the pattern established in [1] with the purpose of analyzing the so-called disjointness-preserving linear maps. This class of maps has been extensively studied in different contexts: Banach lattices, function algebras, and general Banach algebras. A linear map $\Phi: A \rightarrow B$ between Banach function algebras $A$ and $B$ is said to be disjointness-preserving if $\Phi(a) \Phi(b)=0$ whenever $a, b \in A$ are such that $a b=0$. The question of whether such a map for certain algebras is a weighted composition map has been widely studied. This paper focuses on a variety of significant Banach function algebras associated with a locally compact group $G$ such as the Figà-Talamanca-Herz algebra $A_{p}(G)$ and the Figà-Talamanca-Herz-Lebesgue

Copyright 2016 by the Tusi Mathematical Research Group.

Received Oct. 8, 2015; Accepted Jan. 18, 2016.

${ }^{*}$ Corresponding author.

2010 Mathematics Subject Classification. Primary 43A15; Secondary 43A30, 46J10.

Keywords. Fourier algebra, Figà-Talamanca-Herz algebra, Figà-Talamanca-Herz-Lebesgue algebra, operator hyper-Tauberian Banach algebra, disjointness-preserving linear map. 


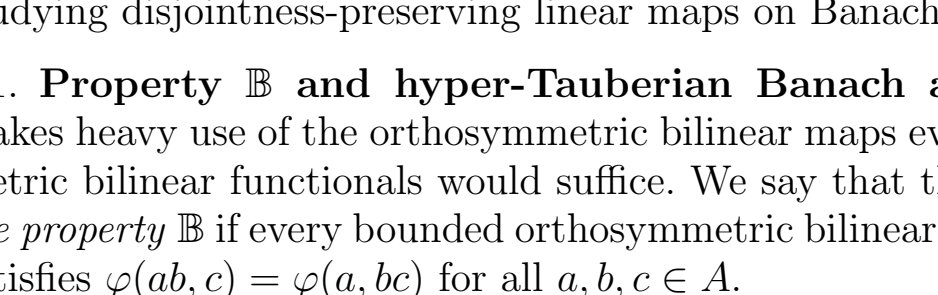

\title{
DISJOINTNESS-PRESERVING LINEAR MAPS ON BANACH FUNCTION ALGEBRAS ASSOCIATED WITH A LOCALLY COMPACT GROUP
}

\author{
J. ALAMinOS, J. EXTREMERA, and A. R. VILLENA* \\ Communicated by K. F. Taylor
}

\begin{abstract}
We introduce a certain property of commutative Banach algebras which we call property $\mathrm{OB}$. We prove that every bounded disjointnesspreserving linear map from a commutative Banach algebra with the aforesaid property to any semisimple, commutative Banach algebra is a weighted composition map. Further, it is shown that a variety of important Banach algebras in harmonic analysis have the property $\mathrm{OB}$.
\end{abstract}

\section{INTRODUCTION}

The basic aim of this article is to bring together the theory of operator hyperTauberian Banach algebras developed by Samei in [15] and the pattern established in [1] with the purpose of analyzing the so-called disjointness-preserving linear maps. This class of maps has been extensively studied in different contexts: Banach lattices, function algebras, and general Banach algebras. A linear map $\Phi: A \rightarrow B$ between Banach function algebras $A$ and $B$ is said to be disjointness-preserving if $\Phi(a) \Phi(b)=0$ whenever $a, b \in A$ are such that $a b=0$. The question of whether such a map for certain algebras is a weighted composition map has been widely studied. This paper focuses on a variety of significant Banach function algebras associated with a locally compact group $G$ such as the Figà-Talamanca-Herz algebra $A_{p}(G)$ and the Figà-Talamanca-Herz-Lebesgue

Copyright 2016 by the Tusi Mathematical Research Group.

Received Oct. 8, 2015; Accepted Jan. 18, 2016.

${ }^{*}$ Corresponding author.

2010 Mathematics Subject Classification. Primary 43A15; Secondary 43A30, 46J10.

Keywords. Fourier algebra, Figà-Talamanca-Herz algebra, Figà-Talamanca-Herz-Lebesgue algebra, operator hyper-Tauberian Banach algebra, disjointness-preserving linear map. 


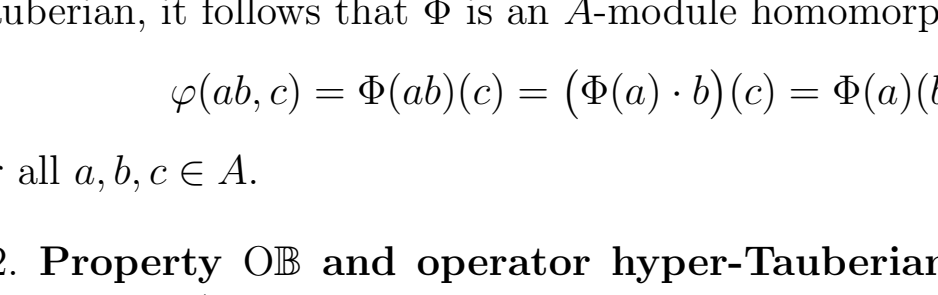

\title{
DISJOINTNESS-PRESERVING LINEAR MAPS ON BANACH FUNCTION ALGEBRAS ASSOCIATED WITH A LOCALLY COMPACT GROUP
}

\author{
J. ALAMinOS, J. EXTREMERA, and A. R. VILLENA* \\ Communicated by K. F. Taylor
}

\begin{abstract}
We introduce a certain property of commutative Banach algebras which we call property $\mathrm{OB}$. We prove that every bounded disjointnesspreserving linear map from a commutative Banach algebra with the aforesaid property to any semisimple, commutative Banach algebra is a weighted composition map. Further, it is shown that a variety of important Banach algebras in harmonic analysis have the property $\mathrm{OB}$.
\end{abstract}

\section{INTRODUCTION}

The basic aim of this article is to bring together the theory of operator hyperTauberian Banach algebras developed by Samei in [15] and the pattern established in [1] with the purpose of analyzing the so-called disjointness-preserving linear maps. This class of maps has been extensively studied in different contexts: Banach lattices, function algebras, and general Banach algebras. A linear map $\Phi: A \rightarrow B$ between Banach function algebras $A$ and $B$ is said to be disjointness-preserving if $\Phi(a) \Phi(b)=0$ whenever $a, b \in A$ are such that $a b=0$. The question of whether such a map for certain algebras is a weighted composition map has been widely studied. This paper focuses on a variety of significant Banach function algebras associated with a locally compact group $G$ such as the Figà-Talamanca-Herz algebra $A_{p}(G)$ and the Figà-Talamanca-Herz-Lebesgue

Copyright 2016 by the Tusi Mathematical Research Group.

Received Oct. 8, 2015; Accepted Jan. 18, 2016.

${ }^{*}$ Corresponding author.

2010 Mathematics Subject Classification. Primary 43A15; Secondary 43A30, 46J10.

Keywords. Fourier algebra, Figà-Talamanca-Herz algebra, Figà-Talamanca-Herz-Lebesgue algebra, operator hyper-Tauberian Banach algebra, disjointness-preserving linear map. 


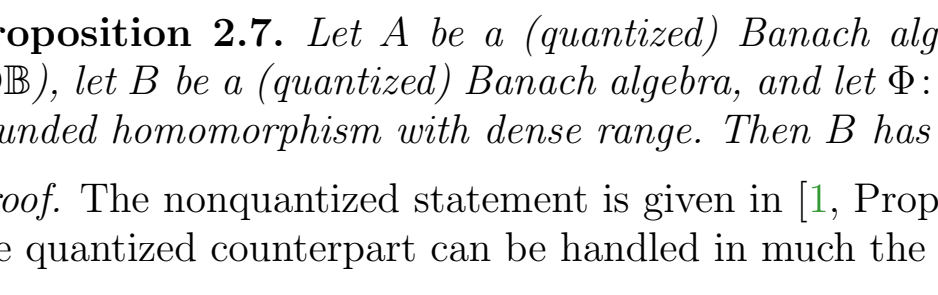

\title{
DISJOINTNESS-PRESERVING LINEAR MAPS ON BANACH FUNCTION ALGEBRAS ASSOCIATED WITH A LOCALLY COMPACT GROUP
}

\author{
J. ALAMinOS, J. EXTREMERA, and A. R. VILLENA* \\ Communicated by K. F. Taylor
}

\begin{abstract}
We introduce a certain property of commutative Banach algebras which we call property $\mathrm{OB}$. We prove that every bounded disjointnesspreserving linear map from a commutative Banach algebra with the aforesaid property to any semisimple, commutative Banach algebra is a weighted composition map. Further, it is shown that a variety of important Banach algebras in harmonic analysis have the property $\mathrm{OB}$.
\end{abstract}

\section{INTRODUCTION}

The basic aim of this article is to bring together the theory of operator hyperTauberian Banach algebras developed by Samei in [15] and the pattern established in [1] with the purpose of analyzing the so-called disjointness-preserving linear maps. This class of maps has been extensively studied in different contexts: Banach lattices, function algebras, and general Banach algebras. A linear map $\Phi: A \rightarrow B$ between Banach function algebras $A$ and $B$ is said to be disjointness-preserving if $\Phi(a) \Phi(b)=0$ whenever $a, b \in A$ are such that $a b=0$. The question of whether such a map for certain algebras is a weighted composition map has been widely studied. This paper focuses on a variety of significant Banach function algebras associated with a locally compact group $G$ such as the Figà-Talamanca-Herz algebra $A_{p}(G)$ and the Figà-Talamanca-Herz-Lebesgue

Copyright 2016 by the Tusi Mathematical Research Group.

Received Oct. 8, 2015; Accepted Jan. 18, 2016.

${ }^{*}$ Corresponding author.

2010 Mathematics Subject Classification. Primary 43A15; Secondary 43A30, 46J10.

Keywords. Fourier algebra, Figà-Talamanca-Herz algebra, Figà-Talamanca-Herz-Lebesgue algebra, operator hyper-Tauberian Banach algebra, disjointness-preserving linear map. 


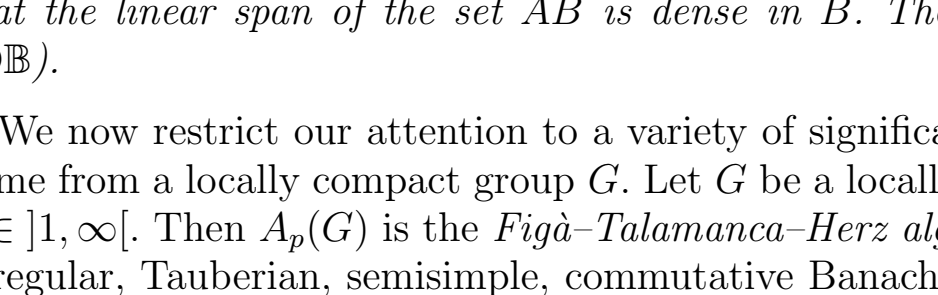

\title{
DISJOINTNESS-PRESERVING LINEAR MAPS ON BANACH FUNCTION ALGEBRAS ASSOCIATED WITH A LOCALLY COMPACT GROUP
}

\author{
J. ALAMinOS, J. EXTREMERA, and A. R. VILLENA* \\ Communicated by K. F. Taylor
}

\begin{abstract}
We introduce a certain property of commutative Banach algebras which we call property $\mathrm{OB}$. We prove that every bounded disjointnesspreserving linear map from a commutative Banach algebra with the aforesaid property to any semisimple, commutative Banach algebra is a weighted composition map. Further, it is shown that a variety of important Banach algebras in harmonic analysis have the property $\mathrm{OB}$.
\end{abstract}

\section{INTRODUCTION}

The basic aim of this article is to bring together the theory of operator hyperTauberian Banach algebras developed by Samei in [15] and the pattern established in [1] with the purpose of analyzing the so-called disjointness-preserving linear maps. This class of maps has been extensively studied in different contexts: Banach lattices, function algebras, and general Banach algebras. A linear map $\Phi: A \rightarrow B$ between Banach function algebras $A$ and $B$ is said to be disjointness-preserving if $\Phi(a) \Phi(b)=0$ whenever $a, b \in A$ are such that $a b=0$. The question of whether such a map for certain algebras is a weighted composition map has been widely studied. This paper focuses on a variety of significant Banach function algebras associated with a locally compact group $G$ such as the Figà-Talamanca-Herz algebra $A_{p}(G)$ and the Figà-Talamanca-Herz-Lebesgue

Copyright 2016 by the Tusi Mathematical Research Group.

Received Oct. 8, 2015; Accepted Jan. 18, 2016.

${ }^{*}$ Corresponding author.

2010 Mathematics Subject Classification. Primary 43A15; Secondary 43A30, 46J10.

Keywords. Fourier algebra, Figà-Talamanca-Herz algebra, Figà-Talamanca-Herz-Lebesgue algebra, operator hyper-Tauberian Banach algebra, disjointness-preserving linear map. 


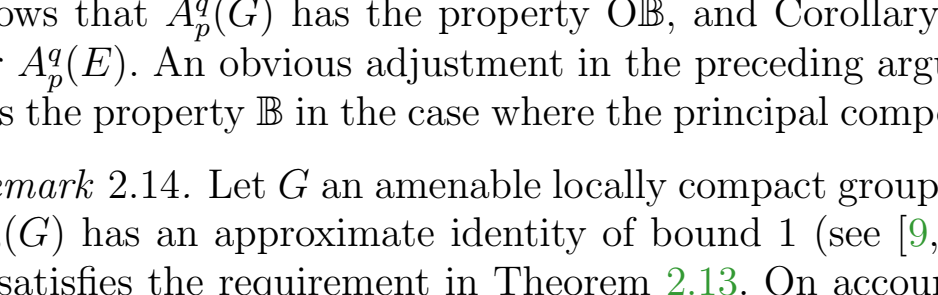

\title{
DISJOINTNESS-PRESERVING LINEAR MAPS ON BANACH FUNCTION ALGEBRAS ASSOCIATED WITH A LOCALLY COMPACT GROUP
}

\author{
J. ALAMinOS, J. EXTREMERA, and A. R. VILLENA* \\ Communicated by K. F. Taylor
}

\begin{abstract}
We introduce a certain property of commutative Banach algebras which we call property $\mathrm{OB}$. We prove that every bounded disjointnesspreserving linear map from a commutative Banach algebra with the aforesaid property to any semisimple, commutative Banach algebra is a weighted composition map. Further, it is shown that a variety of important Banach algebras in harmonic analysis have the property $\mathrm{OB}$.
\end{abstract}

\section{INTRODUCTION}

The basic aim of this article is to bring together the theory of operator hyperTauberian Banach algebras developed by Samei in [15] and the pattern established in [1] with the purpose of analyzing the so-called disjointness-preserving linear maps. This class of maps has been extensively studied in different contexts: Banach lattices, function algebras, and general Banach algebras. A linear map $\Phi: A \rightarrow B$ between Banach function algebras $A$ and $B$ is said to be disjointness-preserving if $\Phi(a) \Phi(b)=0$ whenever $a, b \in A$ are such that $a b=0$. The question of whether such a map for certain algebras is a weighted composition map has been widely studied. This paper focuses on a variety of significant Banach function algebras associated with a locally compact group $G$ such as the Figà-Talamanca-Herz algebra $A_{p}(G)$ and the Figà-Talamanca-Herz-Lebesgue

Copyright 2016 by the Tusi Mathematical Research Group.

Received Oct. 8, 2015; Accepted Jan. 18, 2016.

${ }^{*}$ Corresponding author.

2010 Mathematics Subject Classification. Primary 43A15; Secondary 43A30, 46J10.

Keywords. Fourier algebra, Figà-Talamanca-Herz algebra, Figà-Talamanca-Herz-Lebesgue algebra, operator hyper-Tauberian Banach algebra, disjointness-preserving linear map. 


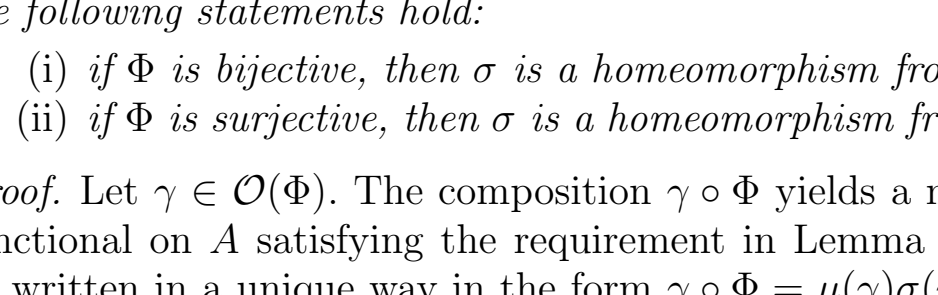

\title{
DISJOINTNESS-PRESERVING LINEAR MAPS ON BANACH FUNCTION ALGEBRAS ASSOCIATED WITH A LOCALLY COMPACT GROUP
}

\author{
J. ALAMinOS, J. EXTREMERA, and A. R. VILLENA* \\ Communicated by K. F. Taylor
}

\begin{abstract}
We introduce a certain property of commutative Banach algebras which we call property $\mathrm{OB}$. We prove that every bounded disjointnesspreserving linear map from a commutative Banach algebra with the aforesaid property to any semisimple, commutative Banach algebra is a weighted composition map. Further, it is shown that a variety of important Banach algebras in harmonic analysis have the property $\mathrm{OB}$.
\end{abstract}

\section{INTRODUCTION}

The basic aim of this article is to bring together the theory of operator hyperTauberian Banach algebras developed by Samei in [15] and the pattern established in [1] with the purpose of analyzing the so-called disjointness-preserving linear maps. This class of maps has been extensively studied in different contexts: Banach lattices, function algebras, and general Banach algebras. A linear map $\Phi: A \rightarrow B$ between Banach function algebras $A$ and $B$ is said to be disjointness-preserving if $\Phi(a) \Phi(b)=0$ whenever $a, b \in A$ are such that $a b=0$. The question of whether such a map for certain algebras is a weighted composition map has been widely studied. This paper focuses on a variety of significant Banach function algebras associated with a locally compact group $G$ such as the Figà-Talamanca-Herz algebra $A_{p}(G)$ and the Figà-Talamanca-Herz-Lebesgue

Copyright 2016 by the Tusi Mathematical Research Group.

Received Oct. 8, 2015; Accepted Jan. 18, 2016.

${ }^{*}$ Corresponding author.

2010 Mathematics Subject Classification. Primary 43A15; Secondary 43A30, 46J10.

Keywords. Fourier algebra, Figà-Talamanca-Herz algebra, Figà-Talamanca-Herz-Lebesgue algebra, operator hyper-Tauberian Banach algebra, disjointness-preserving linear map. 


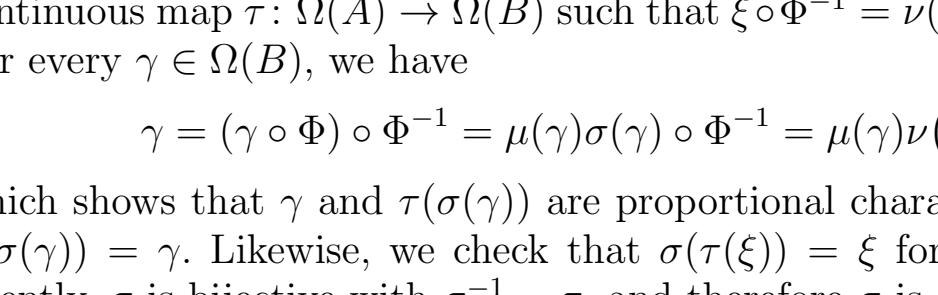

\title{
DISJOINTNESS-PRESERVING LINEAR MAPS ON BANACH FUNCTION ALGEBRAS ASSOCIATED WITH A LOCALLY COMPACT GROUP
}

\author{
J. ALAMinOS, J. EXTREMERA, and A. R. VILLENA* \\ Communicated by K. F. Taylor
}

\begin{abstract}
We introduce a certain property of commutative Banach algebras which we call property $\mathrm{OB}$. We prove that every bounded disjointnesspreserving linear map from a commutative Banach algebra with the aforesaid property to any semisimple, commutative Banach algebra is a weighted composition map. Further, it is shown that a variety of important Banach algebras in harmonic analysis have the property $\mathrm{OB}$.
\end{abstract}

\section{INTRODUCTION}

The basic aim of this article is to bring together the theory of operator hyperTauberian Banach algebras developed by Samei in [15] and the pattern established in [1] with the purpose of analyzing the so-called disjointness-preserving linear maps. This class of maps has been extensively studied in different contexts: Banach lattices, function algebras, and general Banach algebras. A linear map $\Phi: A \rightarrow B$ between Banach function algebras $A$ and $B$ is said to be disjointness-preserving if $\Phi(a) \Phi(b)=0$ whenever $a, b \in A$ are such that $a b=0$. The question of whether such a map for certain algebras is a weighted composition map has been widely studied. This paper focuses on a variety of significant Banach function algebras associated with a locally compact group $G$ such as the Figà-Talamanca-Herz algebra $A_{p}(G)$ and the Figà-Talamanca-Herz-Lebesgue

Copyright 2016 by the Tusi Mathematical Research Group.

Received Oct. 8, 2015; Accepted Jan. 18, 2016.

${ }^{*}$ Corresponding author.

2010 Mathematics Subject Classification. Primary 43A15; Secondary 43A30, 46J10.

Keywords. Fourier algebra, Figà-Talamanca-Herz algebra, Figà-Talamanca-Herz-Lebesgue algebra, operator hyper-Tauberian Banach algebra, disjointness-preserving linear map. 


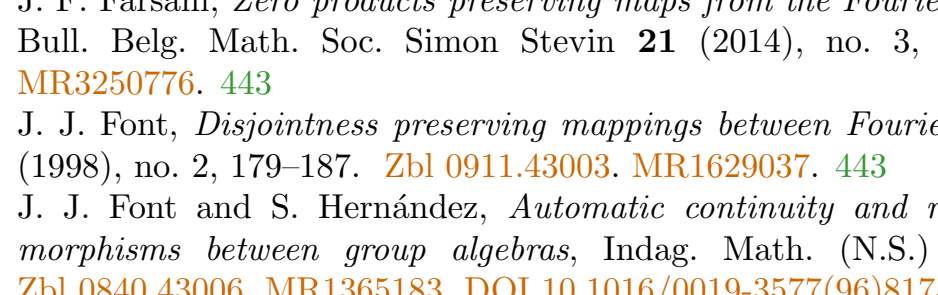

\title{
DISJOINTNESS-PRESERVING LINEAR MAPS ON BANACH FUNCTION ALGEBRAS ASSOCIATED WITH A LOCALLY COMPACT GROUP
}

\author{
J. ALAMinOS, J. EXTREMERA, and A. R. VILLENA* \\ Communicated by K. F. Taylor
}

\begin{abstract}
We introduce a certain property of commutative Banach algebras which we call property $\mathrm{OB}$. We prove that every bounded disjointnesspreserving linear map from a commutative Banach algebra with the aforesaid property to any semisimple, commutative Banach algebra is a weighted composition map. Further, it is shown that a variety of important Banach algebras in harmonic analysis have the property $\mathrm{OB}$.
\end{abstract}

\section{INTRODUCTION}

The basic aim of this article is to bring together the theory of operator hyperTauberian Banach algebras developed by Samei in [15] and the pattern established in [1] with the purpose of analyzing the so-called disjointness-preserving linear maps. This class of maps has been extensively studied in different contexts: Banach lattices, function algebras, and general Banach algebras. A linear map $\Phi: A \rightarrow B$ between Banach function algebras $A$ and $B$ is said to be disjointness-preserving if $\Phi(a) \Phi(b)=0$ whenever $a, b \in A$ are such that $a b=0$. The question of whether such a map for certain algebras is a weighted composition map has been widely studied. This paper focuses on a variety of significant Banach function algebras associated with a locally compact group $G$ such as the Figà-Talamanca-Herz algebra $A_{p}(G)$ and the Figà-Talamanca-Herz-Lebesgue

Copyright 2016 by the Tusi Mathematical Research Group.

Received Oct. 8, 2015; Accepted Jan. 18, 2016.

${ }^{*}$ Corresponding author.

2010 Mathematics Subject Classification. Primary 43A15; Secondary 43A30, 46J10.

Keywords. Fourier algebra, Figà-Talamanca-Herz algebra, Figà-Talamanca-Herz-Lebesgue algebra, operator hyper-Tauberian Banach algebra, disjointness-preserving linear map. 